\title{
AN EXTENSION OF A THEOREM OF GORDON
}

\author{
by E. M. WRIGHT $\dagger$
}

(Received 7 September, 1964)

In what follows all small Latin letters denote non-negative integers or functions whose values are non-negative integers. Let $N=\left(n_{1}, \ldots, n_{j}\right)$ be a $j$-dimensional vector and let $q=q(k ; N)=q\left(k ; n_{1}, \ldots, n_{j}\right)$ be the number of partitions of $N$ into just $k$ parts, each part being a vector whose components are non-negative integers. We write

$$
Q_{j}(k)=Q_{j}\left(k ; X_{1}, \ldots, X_{j}\right)=\sum_{n_{1}, \ldots, n_{j}=0}^{\infty} q\left(k ; n_{1}, \ldots, n_{j}\right) X_{1}^{n_{1}} \ldots X_{j}^{n_{j}}
$$

for the generating function of $q$. We have

$$
F_{J}=\prod_{h_{1}, \ldots, h_{j}=0}^{\infty}\left(1-X_{1}^{h_{1}} \ldots X_{j}^{h_{j}} Y\right)^{-1}=1+\sum_{k=1}^{\infty} Q_{j}(k) Y^{k}
$$

It is well known [3] that

so that

$$
F_{1}=\prod_{h=0}^{\infty}\left(1-X_{1}^{h} Y\right)^{-1}=1+\sum_{k=1}^{\infty} Y^{k} \prod_{s=1}^{k}\left(1-X_{1}^{s}\right)^{-1},
$$

$$
Q_{1}(k)=\prod_{s=1}^{k}\left(1-X_{1}^{s}\right)^{-1}=U\left(X_{1}\right)
$$

(say), but until 1956 the form of $Q_{j}(k)$ for $j>1$ was not known. Carlitz [1] and I [4] showed independently that

$$
Q_{j}(k)=P_{j}\left(k ; X_{1}, \ldots, X_{j}\right) \prod_{i=1}^{J} U\left(X_{i}\right)
$$

(Carlitz dealt only with $j=2$ but this case presents the essential difficulties.) Here $P=P_{j}=$ $P_{j}(k)$ is a polynomial in the $X$, in which no term consists of a power of a single $X_{i}$ only. Thus $P_{1}=1$ but, when $j>1, P_{j}$ is of degree $g=\frac{1}{2} k(k-1)$ in each $X_{i}$, so that

$$
P_{J}=\sum_{h_{1}, \ldots, h_{j}=0}^{g} \lambda\left(h_{1}, \ldots, h_{j}\right) X_{1}^{h_{1}} \ldots X_{j}^{h_{j}} .
$$

Hence, by (1),

$$
q\left(k ; n_{1}, \ldots, n_{j}\right)=\sum_{h_{1}, \ldots, h_{j}=0}^{g} \lambda\left(h_{1}, \ldots, h_{j}\right) \prod_{i=1}^{j} q\left(1 ; n_{i}-h_{i}\right) .
$$

† The research reported herein has been sponsored by the Office, Chief of Research and Development, U.S. Department of the Army, through its European Research Office under contract DA-91-591-EUC-3256. 
In [4] I conjectured that the $\lambda$ are non-negative. Recently Gordon [2] proved this conjecture, essentially by finding the combinatorial interpretation of (2). I have nothing to add to his elegant proof of this result. But he goes on (by a quite different argument) to prove that

$$
P_{j}\left(k ; \xi, \eta, X_{3}, \ldots, X_{j}\right)=0,
$$

where $\xi, \eta$ are primitive $u$ th and $t$ th roots of unity respectively and $1 \leqq u<t \leqq k$. For this purpose he uses a recurrence relation for the $P_{j}(k)$, which both Carlitz [1] and I [4] found.

There is another expression for $P_{j}(k)$, which I found in [4] and which appears at first sight to be rather unpromising. In fact, however, it has proved $[5,7]$ unexpectedly useful to calculate explicit formulae for $q_{j}(k)$ for general $j$ and not too large $k$ and also asymptotic formulae for large $n_{i}$ and all $k$. Recently [6] I found the combinatorial explanation of this expression. Here I use the expression to give an alternative proof of Gordon's result (3) and to take this particular approach to the problem of the form of $P_{j}(k)$ somewhat further.

We write

$$
\beta(m)=\prod_{i=1}^{j}\left(1-X_{i}^{m}\right), \quad \gamma(m)=\prod_{i=1}^{j} \prod_{\rho}\left(1-\rho X_{i}\right),
$$

where $\rho$ runs through all primitive $m$ th roots of unity. Thus

$$
\beta(m)=\prod_{d \mid m} \gamma(d) .
$$

Again $\pi=\pi(k)$ denotes the partition of $k$ into $h(1)$ parts $1, h(2)$ parts 2, and so on, and $\sum_{\pi i(k)}$ denotes summation over all partitions $\pi$ of $k$. Then (6) and (9) of [4] give us

where

$$
P_{j}(k)=\sum_{\pi(k)} \Omega(\pi)
$$

$$
\Omega(\pi)=\left\{\prod_{h=1}^{k} \beta(h)\right\} / \prod_{m}^{k}\left\{h(m) !(m \beta(m))^{h(m)}\right\},
$$

a polynomial in the $X$.

Let $1 \leqq u \leqq k$ and write $v=[k / u]$ and $k=u v+w$, so that $0 \leqq w<u$. We consider separately those partitions $\pi_{1}$ of $k$ which have $v$ parts $u$ and the remaining partitions $\pi_{2}$ in which there are at most $v-1$ parts $u$. We have

$$
P_{j}(k)=\sum_{\pi_{1}} \Omega\left(\pi_{1}\right)+\sum_{\pi_{2}} \Omega\left(\pi_{2}\right)=S_{1}+S_{2}
$$

(say). In the numerator of $\Omega\left(\pi_{2}\right)$, the factor $\gamma(u)$ occurs just $v$ times (once in $\beta(h)$ for $h=u, 2 u$, $3 u, \ldots, v u)$, while it occurs at most $v-1$ times in the denominator. Hence $\Omega\left(\pi_{2}\right)$ has the factor $\gamma(u)$. Thus

$$
S_{2}=\sum_{n_{1}} \Omega\left(\pi_{2}\right)=\gamma(u) T_{2}
$$


where $T_{2}$ is a polynomial in the $X$. Again

$$
\begin{aligned}
S_{1} & =\sum_{\pi_{1}} \Omega\left(\pi_{1}\right) \\
& =(v !)^{-1}\{u \beta(u)\}^{-v} \prod_{h=1}^{k} \beta(h) \sum_{n(w)} \prod_{m}\{h(m) !\}^{-1}\{m \beta(m)\}^{-h(m)} \\
& =(v !)^{-1}\{u \beta(u)\}^{-v} P_{j}(w) \prod_{h=w+1}^{k} \beta(h)=T_{1} P_{j}(w),
\end{aligned}
$$

where $T_{1}$ is a polynomial in the $X$. If $u<t \leqq k$, then $\gamma(t)$ is a factor of $\prod_{h=w+1}^{k} \beta(h)$, but not of $\beta(u)$. Hence $\gamma(t)$ is a factor of $T_{1}$. Thus, if $\xi$ is a root of $\gamma(u)$ and $\eta$ a root of $\gamma(t)$, we have

$$
S_{2}\left(\xi, X_{2}, \ldots\right)=0, \quad S_{1}\left(X_{1}, \eta, X_{3}, \ldots\right)=0, \quad P_{j}\left(k ; \xi, \eta, X_{3}, \ldots\right)=0,
$$

which is Gordon's result.

By a fairly obvious extension of our argument, we find more generally that, if

$$
1 \leqq u_{1}<u_{2}<\ldots<u_{a} \leqq k, \quad v_{b}=\left[k / u_{b}\right], \quad w_{b}=k-u_{b} v_{b},
$$

then

$$
P_{j}(k)=\sum_{b=1}^{a} \frac{P_{j}\left(w_{b}\right) \prod_{h=w_{b}+1}^{k} \beta(h)}{v_{b} !\left\{u_{b} \beta\left(u_{b}\right)\right\}^{v_{b}}}+T \prod_{b=1}^{a} \gamma\left(u_{b}\right),
$$

where $T$ is a polynomial in the $X$.

\section{REFERENCES}

1. L. Carlitz, The expansion of certain products, Proc. American Math. Soc. 7 (1956), 558-564.

2. B. Gordon, Two theorems on multipartite partitions, J. London Math. Soc. 38 (1963), 459-464.

3. P. A. Macmahon, Combinatory analysis, vol. 2 (Cambridge, 1916), 4.

4. E. M. Wright, Partitions of multipartite numbers, Proc. American Math. Soc. 7 (1956), 880-890.

5. E. M. Wright, Partitions of multipartite numbers into a fixed number of parts, Proc. London Math. Soc. (3) 11 (1961), 499-510.

6. E. M. Wright, Direct proof of the basic theorem on multipartite partitions, Proc. American Math. Soc. 15 (1964), 469-472.

7. E. M. Wright, Partition of multipartite numbers into $k$ parts, J. für. Math. 216 (1964), 101-112.

UNIVERSITY OF ABERDEEN 